In discussion, Dr. Burns wondered whether the nerves in clonus might fire almost simultaneously due to hyperexcitability. The observed oscillation frequency corresponds well to a closed-loop oscillation. based on the 100-msec. time-delay to hyperexcitability in a nerve after firing.

In the afternoon, Dr. MacNaughton introduced a patient with Parkinson's discase. The particular symptom studied was a tremor of the thumb and forefinger, in the frequency range $3-5$ c.p.s. Amplitude increased with concentration, excitement and fatigue, but disappeared during sleep. Applica. tion of a heavy weight to the hand only reduced the amplitude. Purposeful action of the hand was quite possible and tremor decreased during a 'slewing' action, but increased, for example, as the hand approached the pen it was going to pick up.

Dr. Blundell (Montreal Neurological Institute) discussed the tremor problem from the point of view of the surgeon, particularly in relation to Parkinson's disease. Surgical possibilities are: (1) To cut the motor nerves; but then no movement is possible. (2) To cut the afferent nerves. This removal of feedback would be justified in extreme cases if it overcame incapacitating tremor. This is true of clonus, but in one known operation on a Parkinsonian case, tremor remained, although at a different amplitude. (3) To cut in the area of the central nervous system. This is the current field of research. Results so far are rather conflicting, and there is insufficient knowledge for confident surgery. Dr. Blundell then detailed the following possibilities as tremor producers: (1) scrvo-loop oscillations ; (2) rhythmic oscillations, for example, by a steady driving mechanism in the brain; (3) hyperexcitability of cells, due to disease.

Dr. Burns also developed the physiology of muscle action somewhat further. Recently a second loop (the 'gamma' loop) has been identified by which 'small' motor neurons are fired from the spinal cord to contract the fibre which is measured by the length receptor. In addition, the afferent nerves from the stretch receptors communicate with the central nervous system. In turn the central nervous system communicates with both the 'big' and 'small' motor neurons. Several more loops are thus added, and much remains to be learned of the combined control action.

Dr. Jasper (University of Montreal) described extensive experimental measurements on tremor, being carried out at the University of Montreal, using micro-electrodes. Frequency measurements of the action potential of muscles in clonus and Parkinson's disease look exactly alike. On the other hand, the cortical phenomena seem different. Some 15-20 per cent of the cortex cells seem to show activity correlated with tremor, but not enough is yet known. In monkeys, tremor has been produced by a small cut in the brain in the appropriate area; a 'large' cut can then remove the tremor.

Finally, Dr. J. A. Tanner (National Research Council) roported the work being done by Massachusetts Institute of Technology and Yale University on measuring the impedance of the wrist and finger extremitics when subjected to sinusoidal or stochastic vibrations. The impedance falls with frequency as might be expected for an inertial load, except that in healthy people there are resonant peaks in the 7 and 17 c.p.s. regions. These are as yet unexplained. In patients with tremor the major resonance is due to the tremor, but at a different frequency than the tremor itself, lying between the 7-17 c.p.s. peaks. This overshadows the other resonances, naturally enough.

A spirited discussion followed concerning the relative usefulness of the 'black box' technique as illustrated by the impedance measurements just described, and the classic analysis-techniques in which individual elements are isolated and their characteristics learned before they are combined together. Everyone agreed that the latter method is ideal if it can be used. Opinion was divided as to whether the 'black box' techniques could be useful, since the 'black box' may often be altogether too complex. I myself foel that both approaches are useful and must be pursued in parallel. This in fact is done of necessity in both engineering and medicine at least.
J. H. Mirsum

\title{
SCHIZOPHRENIA AND LOW-GRADE MENTAL DEFICIENCY IN SWEDEN
}

\begin{abstract}
A CLINICAL and genetico-statistical analysis of schizophrenia and low-grade mental deficiency of a large rural population of 25,000 people in Sweden indicated that altogether 1,790 individuals belonging to 231 families were suffering from schizophrenia, and 1,541 individuals belonging to 215 families were low-grade mental defectives*.

Of the cases of schizophrenia, 50 per cent were classified as hebephrenic, 33 per cent as catatonic, 10 per cent as paranoic and 7 per cent as atypical. 70 per cent of the cases had symptoms generally described as catatonic, but only 20 per cent had thern in connexion with the onset of the psychosis.

* A Clinical and Genetico-Statistical Study of Schizophrenia and Low-Grade Mental Deficiency in a Large Swedish Rural Population. By Bertil Hallgren and Torsten Sjögren. (Acta Psychiatrica et Neuro logica Sicandinarica, Supplementum 140, Vohmen 35, 1959.) Pp. 65. (Copenhagen: Ejnar Munksgaard, 1959.)
\end{abstract}

The mean age at onset of schizophrenia among females, 32 years, is significantly higher than that, 28 years, among males. The psychosis had an acute onset in approximately 40 per cont of the cases and a periodical course in many cases, there being no sex difference. There is thus a positive relationship between acute onset of schizophrenia and periodical course of the psychosis.

There was a statistically significant association between schizophrenia and mental defieiency. Such an association has not been statistically established previously. The morbidity-rate for montal deficiency --including high-grade defectives-among the schizophrenic propositi is $10.5+1.9$ per cent as against a risk of 3 per cent estimated for tho general population. The morbidity-rate for mental deficiency among the sibs of propositi was no higher than the 
risk in the general population of the area studied, nor was the rate of schizophrenia among the sibs of propositi affected with low-grade mental deficiency increased.

The genetico-statistical analysis confirms the conclusions of previous investigations that specific genetic factors are of importance for the manifestation of schizophrenia. The morbidity-rate for schizophrenia as well as that for non-psychotic mental abnormalities among the sibs of propositi was sig. nificantly higher in the families where one of the parents was affected with psychosis or non-psychotic mental abnormality (mainly severe personality deviations) than in those where both parents were unaffected.

Of the mental defectives 88 per cent belonged to the group of uncomplicated, undifferentiated mental deficiency. In $4 \cdot 1$ per cent of the cases the mental defect was combined with epilepsy, in $3 \cdot 3$ per cent with deaf-mutism and in $2 \cdot 6$ per cent with spastic paresis. These figures are considerably higher than corresponding frequencies of epilepsy, deaf mutism and spastic paresis in the general population.

The genetico-statistical analysis confirms the findings of previous investigations that specific genetic factors are of importance for the manifestation of undifferentiated low-grade mental deficiency. In the unclassified material, the morbidity-rate for the lowgrade mental deficiency among the sibs of propositi with uncomplicated undifferentiated mental deficiency is $8.4+0.9$ per cent. This rate is significantly higher than the corresponding risk, 0.9 per cent in the general population. The rate among brothers, $11 \cdot 3 \pm 1 \cdot 5$ per cent, is significantly higher than that among sisters, $5 \cdot 6 \pm 1 \cdot 1$ per cent.

\title{
RADIOACTIVE FALL-OUT IN GHANA
}

\author{
By DR. A. H. WARD and J. D. MARR* \\ Physics Department, University College, Legon, Ghana
}

$\mathrm{R}^{\mathrm{o}}$ OUTINE radioactive survey measurements were started in Ghana in October 1959, after the announcement of French atomic testing plans at Reggan in the northern Sahara. The measurements were extended in January 1960, and the first atomic explosion at Reggan was on February 13. It gave a peculiarly simple variation of radioactive fall-out in Ghana, as it was a single isolated test, after all other fall-out levels had fallen considerably following the cessation of atom bomb tests in 1958. The unexpected relatively large increase in radioactivity of samples of dust, crops, soil and water is summarized ; most of the measurements were by gross $\beta$ - or $\gamma$-counting, and no chemical separation has yet been made.

Radioactivity-levels were very low in Ghana before February 13 ; there is little natural uranium or thorium in the country, and because of Ghana's position near the equator, world-wide fall-out from earlier atomic tests had produced only very slight contamination. Daily measurements since January of activity in air-borne dust, deposited dust, and milk have been made at two laboratories, and the main results are shown in the two graphs. Tamale is in the Northern Region of Ghana, 1,000 miles south of Reggan, and the Accra laboratory is at Legon, 8 miles from the coast and 1,400 miles south of Reggan.

The graphs show the daily measurements of radioactivity in air-borne dust per cu. m., and in deposited dust per sq. m. per day. Air-borne dust is collected by filtration under forced flow, using the method developed by the Radiation Protection Division of the Canadian Department of Health and Welfare; an air pump is fitted with a glass-fibre filter paper and with the average loading of dust has a capacity of about $720 \mathrm{~m}^{3}$ a day. Each pump and filter is in a Stevenson screen, that at Tamale being $3 \mathrm{ft}$. above ground-level, that at Accra being on a flat roof $21 \mathrm{ft}$. above ground-level. Deposited dust was swept up daily from a measured area of flat concrete roof,

* Technical adviser loaned to Ghana by Canadian Government.
$21 \mathrm{ft}$. above ground-level at both Tamale and Accra ; during peak periods the same area was swept each day until any remaining activity was negligible.

The contamination reaching Ghana was dustborne, and from the graphs the bulk of the contamination appears to arrive between the third and tenth days after the explosion. Thereafter, the daily arrival of contamination is seen to fall off slowly; some of the delayed arrival may be 'secondary' in origin, as dust was blowing up freely from all soil and sand surfaces in Ghana and in the Sahara at this time, due to the extremely dry weather conditions. The fall-off in daily arrival of contamination is, however, more rapid than the radioactive decay of mixed fission products, indicating that the total radioactivity carried by this 'secondary' dust is very much less than in the original bulk of contaminated dust direct from Reggan.

The decay of each sample is complicated. All air filters collect natural radium and thorium daughter products as well as fall-out radioactivity. The radium and thorium daughter products decay rapidly, and their effective $\mu \mu \mathrm{c} . / \mathrm{m}^{3}$ content is shown on the graph up to February 16. After this date, the fall-out alone is shown; from repeated counts the contribution of the longer-lived fall-out can be separated from the rapid decay of the radium and thorium daughter products. On the other hand, for deposited dust the total radioactivity content is shown in all cases. Each sample of fall-out has been stored and counted repeatedly to establish its own decay scheme. Apart from some of the 'peak' samples which initially decayed more rapidly, all samples gave a rate of decay fitting closely to the curve :

$$
I_{t}=I_{0} t^{-1.3} \text { (from } t=4 \text { days onwards) }
$$

Dust particles varied in size, and it seemed likely that the larger particles were more local in origin than the smaller ones. Specimens of collected dust were therefore passed through a $50-\mu$ sieve, and it was found that the specific activity of the transmitted 